\title{
Enquête
}

Archives de la revue Enquête

4 | 1988

Varia

\section{Composition romanesque et construction sociologique}

L'anatomie du goût populaire chez Zola

\section{Claude Grignon}

\section{(2) OpenEdition \\ Journals}

Édition électronique

URL : http://journals.openedition.org/enquete/60

DOI : 10.4000 /enquete. 60

ISSN : 1953-809X

Éditeur :

Cercom, Éditions Parenthèses

Édition imprimée

Date de publication : 2 juin 1988

Pagination : $3-25$

\section{Référence électronique}

Claude Grignon, «Composition romanesque et construction sociologique », Enquête [En ligne],

4 | 1988, mis en ligne le 27 juin 2013, consulté le 20 avril 2019. URL : http://journals.openedition.org/ enquete/60 ; DOI : 10.4000/enquete.60

Ce document a été généré automatiquement le 20 avril 2019. 


\title{
Composition romanesque et construction sociologique
}

L'anatomie du goût populaire chez Zola

\author{
Claude Grignon
}

1 Je partirai d'un texte d'Ernest Renan qui devrait nous aider à mieux discerner l'ambiguïté des relations que la sociologie, à la suite de l'histoire, entretient avec la littérature.

«Toute généralisation est attaquable, et la seule manière d'écrire l'histoire qui échappe à la critique est la manière plate qui se borne à d'insignifiantes particularités. Mais que dis-je ? Celle-ci est la plus fausse de toutes, et la prétendue exactitude dont elle est si fière n'est au fond qu'un mensonge. L'imagination, que les historiens exclusivement érudits proscrivent avec tant d'anathèmes, a souvent plus de chances de toucher le vrai qu'une fidélité servile, qui se contente de reproduire les récits originaux des chroniqueurs. Les gravures des ruines de Rome, de Piranesi, sont essentiellement fautives, si on les envisage comme des images de monuments existants: elles changent une foule de détails, elles en ajoutent, elles intervertissent les plans et les distances. Et pourtant, si on les prend, non comme une représentation des traits matériels du paysage, mais comme un essai pour en rendre l'impression générale, elles sont plus exactes que la meilleure photographie: celle-ci, en effet, ne nous montre que des lignes inanimées, elle dissimule l'âme et le type idéal de l'objet qu'elle reproduit, tandis que la gravure en donne le sens moral et esthétique, c'est-à-dire, au point de vue d'une philosophie élevée, sa plus intime réalité [...] L'histoire d'ailleurs est un art autant qu'une science $^{1}$. »

2 Ce texte est lui-même ambigu. On peut, dans une première lecture, y voir une bonne illustration de la démarche propre aux sciences sociales. En opposant la vérité à l'exactitude, Renan nous aide à rappeler aux intégristes du positivisme que l'histoire ou la sociologie ne peuvent pas s'aligner sur l'idée naïve et sommaire qu'ils se font des sciences exactes, qu'il ne suffit pas d'accumuler les «faits» et de décrire, et que du reste toute description engage à sa manière une interprétation, la platitude ne procurant que l'illusion de la neutralité et de l'objectivité. Il montre du même coup que l'historien, ou le sociologue, n'a pas seulement à exposer le résultat de ses recherches, qu'il ne peut pas se 
contenter de rédiger, mais qu'il est bien obligé d'écrire, que cela lui plaise ou non. L'interaction entre les logiques respectives de l'exposé et de la recherche que suggère ce texte correspond beaucoup mieux à la pratique réelle des sciences sociales que la dissociation artificielle des opérations et des étapes; nous commençons en effet à écrire dès le premier commentaire du premier tableau ou de la première interview, ou dès que nous décidons des titres provisoires des dossiers dans lesquels nous rangeons notre "matériel »; et nous n'arrêtons pas de chercher quand nous nous mettons "pour de bon » à écrire. Même si nous avons fait consciencieusement notre travail de documentation et d'enquête, les difficultés que nous rencontrons dans l'expression nous alertent, nous renvoient aux données, nous obligent à imaginer des manières nouvelles de les questionner, appellent des compléments d'information, de nouveaux tris, de nouvelles lectures, de nouveaux rapprochements, de nouvelles comparaisons, etc.

Il serait sans doute exagéré d'assimiler le «type idéal » de Renan à celui de Max Weber ; mais on peut trouver déjà chez lui l'idée que l'interprétation (en l'occurrence la lecture des documents) se fait en fonction de la définition, relative, de l'« essentiel » et de l'«accessoire » que déterminent, pour chaque objet, les intérêts et la problématique de l'historien. "Reconnaître la valeur d'un texte est en un sens le découvrir. Des témoignages sur lesquels l'ancienne critique avait passé avec indifférence sont devenus des traits de lumière. » C'est le point de vue qui crée le «fait »; celui-ci n'existe qu'en fonction de l'intention (qui peut n'être qu'une intuition) théorique qui le rend remarquable:

"Ouvrir une nouvelle série d'aperçus historiques, c'est presque toujours créer une série de documents négligés jusque-là, ou montrer dans ceux qui étaient déjà connus ce qu'on n'avait pas su y voir².»

4 Autre rencontre avec Weber : dire qu'une science, comme l'histoire, est également un art est une manière de reconnaître que la science ne demande pas seulement les qualités que les littéraires concèdent aux scientifiques - la méthode, l'esprit critique, la patience, la minutie, la rigueur et l'humilité -, mais qu'elle exige elle aussi l'imagination et la passion créatrices ${ }^{3}$.

«L'histoire n'est pas une de ces études que l'Antiquité nommait umbratiles, pour lesquelles il suffit d'un esprit calme et d'habitudes laborieuses; elle touche aux problèmes les plus profonds de la vie humaine; il y faut l'homme complet avec toutes ses passions. L'âme y est aussi nécessaire que dans un poème ou une œuvre d'art, et l'individualité de l'écrivain doit s'y refléter. »

5 Mais on trouve aussi dans ce texte un portrait de l'historien en écrivain romantique, une célébration de l'art et une humiliation de la science. Augustin Thierry, qui sert de modèle à Renan, n'est pas seulement un parfait écrivain, qui pousse le souci de la forme et «le soin du style à un degré incomparable ", et qui "affectionne le plus cette humble partie du travail littéraire, qui consiste surtout à éteindre et à effacer ", quitte à ne dicter que " quinze à vingt lignes par jour »; c'est un " grand artiste», un peintre, qui sait « rendre la vie aux générations éteintes ». Du génie romantique, il a tous les dons et tous les signes: l'inspiration, l'imagination, la passion, l'intuition, la vocation, la solitude, la sensibilité, l'enthousiasme qui le pousse à s'engager dans les luttes politiques et idéologiques de son temps. Visionnaire, véritable prophète du passé, A. Thierry puise «dans le vif sentiment de son époque et l'ardeur de sa propre passion [...] le souffle fécond qui, passant sur le champ des morts, comme dans la vision d'Ezéchiel, leur rendait la vie, et d'ossements épars faisait des hommes "; il « ressent d'une manière personnelle les joies et les douleurs des hommes d'autrefois », il a l'« intuition directe des sentiments 
et des passions du passé ", le "sens historique s'est manifesté en lui spontanément et comme par une sorte de révélation $»^{4}$. Du côté de l'art (derrière lequel on devine sans peine la religion), Renan range tout ce qui lui paraît sacré et sublime, le "génie créateur", le "génie supérieur», la "haute intelligence», la "gloire souveraine d'inventer ", Chateaubriand, la "lutte héroïque d'une âme forte", une "vie presque miraculeuse ", l'« amour ", la « foi », etc.; et, à la suite d'A. Thierry, il ramène la science, réduite à " la recherche et la discussion des faits, sans autre dessein que l'exactitude ", au rang d'un savoir technique, nécessaire mais subalterne; il l'associe, au mieux, aux qualités mineures, aux « humbles avantages », aux « habitudes laborieuses ", à «l'étude consciencieuse", à «la menue recherche du détail», au pire à "la démonstration littérale », à la « sécheresse des faits », à la " platitude » et à « l'insignifiance ». D'un côté, la souveraineté des "grands créateurs » et des "maitres », que la critique " ne saurait atteindre », de l'autre des troupes anonymes d'érudits : la « vieille école » des bénédictins (que tout oppose à la "grande école » à laquelle appartient A. Thierry) «a pour mission de fournir les matériaux de l'histoire, mais non de construire l'histoire elle-même »; elle "pose la condition de l'histoire", mais elle ne la fait pas; quant à la "seconde génération » des " docteurs ès lettres » et des " agrégés », elle doit tout aux fondateurs, y compris la capacité de « relever des fautes dans leur œuvre $»^{5}$.

6 En fait, Renan conçoit la vérité en histoire sur le modèle de la vérité dans l'art; il n'oppose le vrai à l'exact que parce qu'il confond ici, dans le même mouvement, le vrai avec le beau'. Ce n'est pas un hasard s'il assimile les « fautes de jugement » à des «fautes de goût ", s'il considère que la "pensée n'est complète que quand elle est arrivée à une forme irréprochable, même sous le rapport de l'harmonie $»^{7}$, ou s'il prend l'exemple des gravures de Piranèse. Du point de vue de la peinture, l'exactitude du détail peut ne pas importer à la vérité de l'ensemble; si l'on prend parti contre le «fini » ou le «léché » (question de mots, question de goûts), on dira même que l'ensemble paraît d'autant plus vrai que le détail est plus flou et plus négligé. Assimiler l'histoire à un art conduit à tourner le dos aux instruments et aux procédures de la vérification scientifique et à leur substituer les critères esthétiques et mondains du « naturel » et de la « vraisemblance ». Il n'est pas étonnant que Renan refuse aux savants le droit de se prononcer en dernier ressort sur la valeur de leur œuvre pour le réserver aux «gens du monde, excellents juges, non du travail scientifique nécessaire pour la connaissance des faits, mais de la vérité générale $d u$ récit $»^{8}$. Notons quand même que cette abdication du jugement scientifique est incompatible avec l'exigence durkheimienne de la rupture avec les prénotions: n'a des chances de passer pour vrai, dans cette perspective, que ce qui confirme et corrobore le sentiment public et le goût dominant.

7 L'attraction de la littérature s'exerce sans doute moins fortement sur la sociologie que sur l'histoire, qui est elle-même un genre littéraire consacré ; on ne parle pas, ou du moins pas encore, de « roman sociologique » comme on parle de roman historique. Mais on peut se demander si certaines des branches de la sociologie, en particulier la sociologie des goûts ou des "styles de vie », ne sont pas en train de prendre la place laissée vacante par le roman de mœurs, après que les romanciers (ou du moins leur avant-garde) en aient abandonné la formule. Par le sérieux de la documentation et de «l'enquête », les romans réalistes de G. Flaubert nous mettent au défi de distinguer entre la vérité littéraire et la vérité dans les sciences sociales. Ce qui paraît littérairement vraisemblable peut pourtant être statistiquement et sociologiquement improbable; ainsi Emma Bovary, «femme, jeune, mariée, mère d'un enfant, rurale et catholique, cumule les traits dont Durkheim a 
montré qu'ils constituaient les facteurs les plus efficaces de préservation du suicide $»^{9}$. Le destin de Frédéric Moreau, dans L'éducation sentimentale n'est pas moins paradoxal : c'est au moment où il réunit toutes les chances et tous les facteurs de la réussite (fortune, relations, beau mariage en vue), que son ratage définitif se noue et se précipite. Mais, par ailleurs, il suffit de faire une coupe synchronique dans Madame Bovary ou dans L'éducation sentimentale, de suspendre l'action, de neutraliser le récit, pour obtenir un schéma qui ressemble beaucoup à ceux de nos analyses factorielles des correspondances, et qui se laisserait aisément commenter à la manière de $L a$ Distinction, si l'on veut bien me permettre cet hommage à Paul Reboux. Ainsi tout, de son hôtel particulier à sa bibliothèque "de jeune fille ", à son coupé, ou aux restaurants chics qu'il fréquente (Le Café anglais, Véfous) dit la distance sociale qui sépare Frédéric Moreau (vingt-sept mille livres de rentes) de son ami Deslauriers, «le clerc», "le cuistre», "l'avocat» (sans causes), avec " son mauvais paletot de lasting ", "sa pauvre redingote ", " ses restaurants à trente-deux sous", ses "voyages en omnibus " et l'ouvrière qui lui tient lieu de maîtresse ; un autre système de différences oppose le luxe tapageur de la « constellation Arnoux » (à l'intersection de la bohème et de la spéculation) à l'ambiance feutrée, austère et raffinée de la « constellation Dambreuse », que sa "multipositionnalité » dans la haute finance et dans la haute politique, et sa double appartenance à l'aristocratie et à la grande bourgeoisie placent au sommet de la pyramide sociale. Par un effet de "dominocentrisme" qu'on retrouve dans les cartes que dessinent les explorations « légitimistes » de l'espace social, la description s'appauvrit à mesure qu'on va des classes supérieures aux classes moyennes, et des classes moyennes vers les classes populaires ${ }^{10}$ : l'anatomie flaubertienne des goûts s'organise, déjà, autour de l'opposition entre la foule anonyme et indifférenciée et l'individu, entre le peuple, présent à l'arrière-plan, sur la toile de fond historique, mais qui ne participe pas à l'action romanesque proprement dite, et les protagonistes, qui appartiennent tous, par leurs positions, leur origine ou au moins leur trajectoire sociale, aux classes supérieures ou aux classes moyennes ${ }^{11}$. Ainsi, tout se passe comme si l'art du romancier se réfugiait dans l'écart entre le destin social correspondant aux propriétés sociologiques dont il dote ses personnages, et le destin improbable qu'il leur ménage. Plus les personnages sont importants, plus la description de ces propriétés est fouillée et minutieuse, et plus cet écart est grand ; c'est précisément son amplitude qui individualise le personnage du roman et qui le propulse au premier plan, qui le transforme en héros en en faisant un être d'exception, échappant à la règle commune et par là même "intéressant». Comme son héroïne, Flaubert «se tue » au «travail du style » en voulant à tout prix faire sortir le merveilleux de l'ordinaire. Emma ne l'intéresse, et il ne sympathise avec elle, que dans la mesure où les insatisfactions et les provocations qu'il lui prête lui permettent de composer avec le pensum prosaïque imposé par Bouilhet et Du Camp ; en imaginant son destin improbable et son agonie romantique, il se revanche du labeur et de la mortification imposés par la peinture réaliste de la scène des Comices. Ainsi le réalisme quasi sociologique de Flaubert sert à la fois d'alibi, de repoussoir et de tremplin à la fantaisie et au romanesque : à partir d'un fait-divers qui présente toutes les garanties de la banalité, et sous couvert d'étudier un cas concret, il forge un type idéal, qui s'oppose trait pour trait au type moyen, mais qu'il rend vraisemblable à force de minutie, et qu'il parvient à faire passer pour un cas typique ${ }^{12}$. Pour reprendre la formule de J.C. Chamboredon, un hapax finit par représenter un genre ; peut-être touchons-nous là un des ressorts essentiels de ce mystérieux "effet de réel » qui a été si souvent invoqué durant ces journées. 
8 L'ambiguïté des relations que les sciences sociales entretiennent avec l'écriture littéraire se voit sans doute encore mieux lorsque les romanciers se risquent à choisir leur héros dans les classes populaires. Ce n'est pas, comme on pourrait le croire en prenant au pied de la lettre leurs déclarations de principe ${ }^{13}$, parce que les naturalistes se sont voulus les liquidateurs du roman romanesque et les fondateurs du roman «scientifique " et « expérimental » qu'ils sont les précurseurs des sociologues. Confrontés à des problèmes analogues, du moins dans leur principe, à ceux que rencontre la sociologie lorsqu'elle entreprend d'étendre ses analyses aux classes populaires (comment donner une forme savante au témoignage vécu, au document brut, sans en altérer la saveur? Comment faire passer le parler populaire, langue orale par excellence, dans cette langue doublement écrite qu'est la langue littéraire ?, etc.) ${ }^{14}$ les naturalistes ont perfectionné les procédés qui permettent de produire dans le même mouvement l'illusion de l'exactitude et l'impression de la vérité. Dans L'Assommoir, par exemple, la démonstration repose pour l'essentiel sur une succession de tableaux qui sont autant de mises en scène de la vie quotidienne des classes populaires. Pour donner à penser, Zola s'efforce de donner à voir: il sélectionne, en fonction des besoins de l'intrigue et de la thèse, un certain nombre de comportements, d'interactions, bref de «traits » qu'il juge à la fois représentatifs et typiques, et qui lui paraissent propres à illustrer son propos d'une manière exemplaire. Par exemple, la danse de Gervaise recevant ses vingt-cinq francs du mont-de-piété illustre l'imprévoyance et l'infantilisme des ouvriers ; Goujet forcé d'accepter une tournée, Goujet «s'emplissant trop» et "se piquant le nez» illustre le danger des mauvaises fréquentations, le caractère pathogène du milieu; les bourrades amicales et la réconciliation qui suivent l'altercation entre Coupeau et Lantier illustrent l'alternance caractéristique (pour le point de vue dominant) de la violence et de la veulerie populaires. La technique du romancier est très proche, sur ce point, de celle du sociologue qui fait de la «description en concepts » et qui s'efforce que l'analyse se dégage pour ainsi dire d'elle-même de l'observation des faits et de la présentation du matériel (comme le fait, par exemple, Erving Goffman dans Asiles ) - à ceci près que, chez l'écrivain, la sélection des traits pertinents se fait de droit, d'une manière arbitraire, en fonction du parti-pris de départ qui préside à sa conception de l'œuvre et qui gouverne l'ensemble de ses choix ultérieurs ${ }^{15}$.

9 Par ailleurs, Zola étend au niveau de la description et du constat le pouvoir discrétionnaire d'intervention que le romancier réaliste exerce sans complexe au niveau de l'intrigue et de la fiction. À la suite des Goncourt, il se propose d'exploiter au niveau du peuple la veine réaliste; il entend bien se démarquer des représentations édifiantes et bêtifiantes de l'ouvrier vertueux qu'on trouvait, entre autres, chez Eugène Manuel ou chez Arsène Houssaye ${ }^{16}$. L'Assommoir sera donc un tableau à la fois « effroyable » et «très exact » de la vie de l'ouvrier ; Zola veut décrire le peuple tel qu'il est, « avec ses ordures, sa vie lâchée, son langage grossier », « montrer le milieu peuple et expliquer par ce milieu les mœurs peuple ", faire voir que "la soûlerie, la débandade de la famille, les coups, l'acceptation de toutes les misères vient des conditions mêmes de l'existence ouvrière, des travaux durs, des promiscuités, des laisser-aller, etc. ${ }^{17}$. Mais le pessimisme de circonstance de Zola s'attelant à son "roman-ouvrier » est si marqué, son parti pris si apparent, que L'Assommoir est presqu'un roman édifiant à l'envers, le vitriol remplaçant l'eau de rose. Comme dans une tragédie classique (ou comme dans Le train de 8 heures 47), l'intrigue est réglée par une "mécanique du contretemps » qui ne laisse aucune chance aux personnages : Coupeau ne peut pas ne pas tomber du toit, Gervaise ne peut pas ne pas 
"se tromper de mari »; bien avant de commencer à rédiger, Zola a décidé de lui "prêter le désir très modeste de ce qu'elle n'aura pas »; « chacune de ses qualités tournera contre elle »; il « la tuera » à quarante et un ans, en 1869, "dans un drame », " tragiquement ", "après l'avoir fait passer par toutes les crises et toutes les hontes imaginables ${ }^{18}$. Lorsqu'il écrit L'Assommoir, Zola exécute fidèlement les consignes qu'il s'est données dans son « Ébauche »; témoin le soin avec lequel il replâtre, pour mieux la profaner, la vertu de sa Justine de la Goutte d'Or: tout ce qui symbolise, au début du repas, l'ordre et la pureté semble n'avoir été mis là que pour être chamboulé, avili et salopé. L'alignement impeccable du couvert appelle immanquablement sa "débandade", de même que la nappe et les rideaux de mousseline attirent par avance les taches et les souillures; la parodie nuptiale par laquelle commence le récit du gueuleton («ça faisait comme une chapelle au milieu de la boutique ») ne demande qu'à s'inverser, à dégénérer en "noce » crapuleuse, en «noce» noire qui inaugure le ménage à trois dans lequel Gervaise va s'enfoncer.

10 En multipliant opportunément et systématiquement les rencontres et les coïncidences, non plus seulement entre les événements ou entre les personnages, mais entre les « petits faits vrais » qu'il accumule et dont il truffe le récit, Zola confère la valeur de symbole à des notations qui, prises isolément et dans le désordre, n'auraient qu'un statut documentaire ou simplement anecdotique. C'est ainsi que la rencontre, un lundi, dans la boutique d'une blanchisseuse, sur la nappe trop blanche d'une table improvisée, d'une surabondance de nourritures et de boissons coûteuses représente la paresse, la voracité et l'imprévoyance ouvrières. La mise en image ne se distingue pas de la mise en scène; la métaphore naît de la description, l'image littéraire de la circulation organisée, à l'intérieur du récit, des images concrètes (visuelles, sonores ou olfactives). C'est de cette manière que Zola établit une correspondance symbolique, à la fois fantastique et vraisemblable, plus suggestive et plus convaincante que toutes les démonstrations logiques, entre la privation de nourriture et la profusion de nourritures: le saladier inépuisable de L'Assommoir, (« là-dedans, on pêchait des morceaux de veau; et il y en avait toujours ») correspond au gouffre sans fond des estomacs populaires, de même que le fonctionnement continu et inexorable de l'alambic correspond à la soif inextinguible des ouvriers. L'association des idées se fait sans qu'on y pense, par le biais de la combinaison des «faits» et des images, et par celui de l'association des images entre elles. À la différence de Denis Poulot, son «informateur ", Zola n'a pas besoin de démontrer la relation entre l'intempérance, la paresse et la déchéance ${ }^{19}$, il lui suffit de faire travailler l'imagination du lecteur en assombrissant progressivement le tableau de la «noce », en associant la montée de la "rigolade » et des clameurs à la tombée de la nuit, bref, en passant du « régime diurne » au « régime nocturne » des images. On pourrait multiplier les exemples de ce recours quasi permanent à l'hypotypose, qui permet à Zola (comme à Flaubert), de faire image à partir de son "matériel », et qui est, chez les Naturalistes, l'instrument par excellence du passage du réalisme à l'imaginaire, du constat à l'interprétation ${ }^{20}$.

11 La prédilection des Naturalistes (Zola, mais aussi les Goncourt et J. K. Huysmans) pour le thème de la ripaille populaire s'explique sans doute par le fait qu'il leur redonne une matière là où la matière fait défaut. Prendre la cuisine et le repas comme motif (au sens où l'entendent les peintres) permet de brosser des scènes de genre typiques, de camper des personnages et de rapporter des propos hauts en couleur, d'énumérer et de nommer à l'envi des objets, des ustensiles, des aliments, des mets, des recettes, bref de remplir le 
récit d'images, et de "faire sensation" en évoquant une infinité de substances, de saveurs, de couleurs, de bruits et d'odeurs. Plus curieux, moins attristant que le meurtde-faim, l'ouvrier noceur est plus facile à peindre; ses extravagances, sa frime, ses prouesses réelles ou légendaires, la verdeur de son langage lui confèrent un rendement expressif bien supérieur. Ainsi la scène du gueuleton permet à Zola d'avoir, dans son " roman ouvrier ", un équivalent vraisemblable des descriptions de repas aristocratiques ou bourgeois qu'on trouve chez Flaubert ou chez Balzac; elle lui donne la possibilité technique de produire, à propos des classes démunies, des développements aussi fournis, des tableaux aussi riches et aussi pleins que ceux auxquels se prête naturellement la diversité inépuisable des propriétés, des manières et des goûts des classes dominantes ${ }^{21}$.

Ainsi la fidélité au réel paraît d'autant plus grande que la documentation est plus légère et plus sélective : la réalité s'intègre d'autant plus facilement et d'autant plus intimement à la fiction que les faits susceptibles de contrarier l'inspiration et la « thèse » de l'écrivain ont été plus souverainement écartés et refoulés. Comme dit Renan, la composition du récit "exige plus d'un parti pris»; l'historien, ou le sociologue-écrivain doit savoir « dépenser son temps et son labeur pour pondérer les parties, construire un ensemble harmonieux avec des matériaux barbares, ici maigres, là surabondants ${ }^{22}$ » (ce qui revient à dire que "l'écriture " peut n'être qu'un cache-misère). Dans le même temps, la réintroduction, sous le couvert de la présentation réaliste des faits, des procédés usuels de l'imagerie littéraire (sous la forme exacerbée que leur a donnée le Romantisme) confère aux « constats » du romancier une puissance d'évocation et un "charisme » sans commune mesure avec ceux auxquels peut prétendre le compte rendu d'une observation scientifique; l'ambivalence et la labilité constitutives des images, qui permettent de dire à la fois le plein et le manque, le rose et le noir, autorisent en souplesse tous les renversements du pour au contre et tous les glissements de sens. Ainsi le libre usage de la métaphore dispense Zola de la reproduction littérale des lieux communs qu'il se propose de romancer : l'évocation des propriétés symboliques que l'opinion dominante prête aux «basses classes» se fait indirectement, par le biais de la description des propriétés matérielles et substantielles des nourritures populaires : Goujet, l'ouvrier vrai, est « doux et solide » comme l'épinée de cochon ; c'est un « sucre ", une "crème ", un «beurre ", tandis que Coupeau est brutal et ravageur comme " l'incendie » que " cette bougresse de blanquette vous met dans le ventre", violent et lâche comme le "vitriol » du Père Colombe ${ }^{23}$.

Bien que Zola soit plus facile à prendre en flagrant délit d'ethnocentrisme de classe qu'un sociologue moderne, c'est sans doute chez lui qu'il faut également chercher le perfectionnement des procédés par lesquels l'écrivain, qu'il soit romancier ou sociologue, parvient à donner l'illusion du relativisme et de la neutralité. La représentation que Zola se fait du peuple et qu'il en donne, le point de vue qu'il prend sur lui et qu'il lui prête, sont sans doute déterminés bien davantage par son ambition littéraire et par le plan de son œuvre à venir que par la familiarité ou l'observation méthodique ; s'il est vrai que Zola a côtoyé le peuple pendant ses années de bohème pauvre, il est étranger, par son origine, par ses études et par ses aspirations, aux milieux populaires qu'il se propose de décrire, et qu'il n'a ni le temps ni le goût de connaître de l'intérieur ${ }^{24}$. C'est en fonction de son programme préalable que Zola fixe à l'avance la place que le peuple occupera dans les Rougon-Macquart et le rôle qu'il y jouera : l'idée littéraire du peuple qu'il forme à partir des humeurs et des préjugés liés à sa position et à sa trajectoire sociale personnelles est inséparable de l'idée volontariste qu'il se fait de son œuvre future, de son ambition, de 
son égocentrisme et de son " furieux appétit de gloire »" Pour « enfoncer» Balzac, son sur-moi, en couvrant l'ensemble des «mondes » sociaux, et pour écraser Flaubert et les Goncourt, ses devanciers et ses modèles, sous le volume et sous la masse de son œuvre « à lui $»^{26}$, il lui faut au moins un ou deux « romans ouvriers» (comme il lui faut un « roman sur l'art» et un autre "sur les prêtres » ${ }^{27}$. De même qu'il a besoin d'une "théorie scientifique » et d'un "système » ad hoc, capables de conférer à son œuvre cohérence et surtout originalité, Zola a besoin d'ouvriers ad hoc: il les trouvera sans peine, chez Denis Poulot ou chez Francisque Sarcey, comme il a trouvé la théorie de l'hérédité chez le Docteur Lucas ${ }^{28}$. Par delà les sources immédiates de L'Assommoir, le thème de la ripaille et de la «rigolade » populaires se rattache à la tradition du pittoresque social. Les «bons zigs » de D. Poulot, «l'ouvrier rigoleur» de F. Sarcey, et avant eux, l'ouvrier «noceur, gouapeur, [...] faisant de sa vie un lundi » que personnifie le Médéric Gautruche de Germinie Lacerteux, correspondent à des stéréotypes bien établis qui renvoient à des faits d'opinion, c'est-à-dire à des faits de l'opinion dominante; on ne les rencontre pas seulement dans la rue, dans les bistrots, dans les bals ou dans les guinguettes, mais aussi, et peut-être surtout, dans la littérature, dans la presse et dans l'estampe ${ }^{29}$. Mais, comme en témoigne le «Dossier » préparatoire de L'Assommoir, cet intérêt pour le pittoresque populaire n'exclut ni la malveillance, ni le mépris de classe: "Toute la sottise des ouvriers dans des conversations. Montrer le manque d'instruction, les préjugés de portières, le milieu déplorable "; "conversation générale bête, pleine de préjugés »; "l'amour de l'argent primant tout chez le peuple »; « Coupeau pas d'instruction ; Lantier de la fausse instruction"; "on n'a les ouvriers que quand ils n'ont plus le sou»; «Lantier, l'ouvrier en paletot, refaisant la carte de l'Europe »; « Lantier épateur. Les gros mangeurs, les gros buveurs et les fiers-à-bras admirés », etc. ${ }^{30}$. Témoin encore le portrait de l'ouvrier que Zola trouve chez F. Sarcey:

«La plupart des ouvriers parisiens sont des rigoleurs et quand ils ont vingt francs en poche, ils n'ont rien de plus pressé que de festoyer jusqu'à la complète et définitive extermination de la pièce jaune [...] J'ai eu des peintres chez moi, et j'étais stupéfait de voir comment ils se moquaient de leur besogne. Ils apportaient leur pot à colle, donnaient quelques coups de pinceau en chantant, s'en allaient déjeuner et ne revenaient plus [...] Avec tout cela rigoleurs et imprévoyants, tout entiers, comme les sauvages et les singes, à la sensation présente. Le bruit d'un écu sonnant dans leurs poches excitait chez eux toutes sortes d'envies qu'ils n'étaient pas maîtres de contenir ${ }^{31}$.»

En dépit des précautions de Zola, cet ethnocentrisme de classe spontané affleure encore en maint endroit sous la neutralité et l'objectivité voulues de L'Assommoir. Témoin la manière dont Zola, dans la scène du gueuleton, se moque des noceuses mises comme des "dames", "sanglées dans leur corsage ", "les bandeaux empâtés de pommade», et l'ironie appuyée avec laquelle il leur donne cérémonieusement du «madame » ou de la «dame » toutes les fois qu'il leur fait commettre des fautes de goût et de savoir-vivre: drôles de dames, qui enlèvent leur corset, grattent des os, tapent sur leur verre, et veulent toutes de la carcasse, parce que «la carcasse, c'est le morceau des dames». Confrontés successivement aux bonnes manières, au goût dominant, et à la culture savante, qui se trouvent du même coup constitués en culture et en goût de référence, les personnages de L'Assommoir se conforment sans le savoir et par anticipation à la théorie de la légitimité culturelle. Avant d'emmener la noce de Gervaise au "Moulin d'Argent ", où elle étale à la fois son manque de savoir-vivre et son excès d'appétit, Zola, par une trouvaille de dernière minute ${ }^{32}$, l'entraîne au musée du Louvre où elle étale son inculture : "Des siècles d'art passent devant l'ignorance ahurie» des malheureux, qui ne 
comprennent rien "à la sécheresse fine des Primitifs ", aux "splendeurs des Vénitiens ", à la « vie grasse et belle de lumière des Hollandais ", et qui s'extasient devant les parquets luisants qu'ils piétinent de leurs gros souliers à clous; Coupeau trouve que la Joconde ressemble à une de ses tantes; Boche et Bibi-la-Grillade « ricanent en se montrant de l'œil les femmes nues », et cherchent "les détails orduriers » dans les Rubens. Pour venir au Louvre, la noce a dû défiler à pied sous la pluie, des quartiers populaires du Nord vers le centre, sous l'œil ironique des passants et des boutiquiers qui contemplent le "décrochez-moi-ça du luxe des pauvres", "les effilés de Mme Lerat ", "la redingote luisante » de Coupeau, le pantalon « jaune canari » de Boche, le « violet cru » de la robe de Mme Gaudron, avec «son ventre de femme enceinte, qu'elle porte énorme, très en avant», "les riflards lamentables», «les vieux chapeaux aux formes pleines de comique ", etc.

15 Pas d'expérience vécue de la condition populaire, pas de familiarité avec la langue et la culture du milieu, peu d'observation, peu d'érudition, l'anti-ouvriérisme exacerbé des lendemains de la Commune : il n'est pas étonnant que L'Assommoir nous renseigne moins sur les mœurs réelles des ouvriers parisiens du Second Empire que sur les humeurs réactives que les relations avec les classes populaires engendrent chez les dominants ou dans les classes moyennes : récriminations de maître ou de client jamais assez bien servis, courroux de patron, dépit de petit rentier ou de petit fonctionnaire devant l'étalage de la " prospérité » populaire, hargne morose de professeur porté à voir partout le chahut et la " rigolade ", et, plus généralement, intolérance de classe épidermique à l'égard d'une «populace » qu'on est malheureusement forcé de côtoyer. Et pourtant L'Assommoir « fait peuple » et "sonne peuple»; Zola réussit à choquer profondément et durablement le goût dominant (et pas seulement le goût et le moralisme étroit de son époque). Ce que ne peut lui pardonner F. Brunetière n'est sans doute pas tant d'avoir abordé des sujets vulgaires que de les avoir traités de telle manière que leur vulgarité rejaillisse sur l'œuvre littéraire en atteignant celle-ci dans sa forme ${ }^{33}$. En effet Zola ne se contente pas de donner les mœurs et les mots du peuple en spectacle: dans la mesure où il s'efforce de les rapporter tels quels, dans leur " authenticité ", il leur accorde une existence autonome et indépendante, à la réalité de laquelle le lecteur cultivé se trouve confronté d'une manière à la fois insidieuse et brutale. La principale trouvaille de L'Assommoir est sans doute l'extension au parler peuple du style indirect libre (couramment utilisé par Flaubert) ${ }^{34}$; la remontée de l'argot, qui comme le remarque F. Brunot, passe du dialogue au récit, de la bouche des personnages à celle de l'auteur, n'a pas seulement pour effet d'encanailler la phrase ; en reprenant à son compte des mots « peuple» (sinon vraiment populaires), Zola amène le lecteur cultivé à parler et à penser peuple, à voir le peuple et à se voir lui-même avec les yeux du peuple (ou du moins avec les yeux que Zola prête au peuple) ) $^{35}$. Ainsi l'épisode de la visite au Louvre semble appeler une autre lecture, « relativiste » celle-là. À l'intrusion de la noce dans le musée correspond l'irruption des mots du parler " peuple » dans la phrase littéraire ; en se mettant à parler comme ses personnages, Zola chahute le lecteur cultivé en l'obligeant à voir les peintures comme il les leur fait voir, à ressentir les impressions naïves qu'il leur fait éprouver, à être, comme eux saisi de vertige devant « ce violent tapage de couleurs ", cette " débandade de gens et de choses ", " ces paysages tout noirs ", " ces bêtes devenues jaunes ", etc. Plongé sans ménagement au sein de la noce, celui-ci se trouve atteint, sinon do cécité, du moins de diplopie culturelle: en même temps que « les petits dieux de l'Orient qui dorment dans les vitrines », il finit par voir, lui aussi, « des bonshommes très laids » et en vient presque à se demander si les dessins des 
maîtres ne sont pas simplement «des feuilles de papier gribouillées, sous les vitres, contre les murs ».

Bien qu'il puisse toujours compter sur l'ignorance de classe du lecteur cultivé, prêt à se faire refiler des blagues de rapins pour des naïvetés populaires authentiques, Zola ne parviendrait sans doute pas à donner aussi fortement l'impression qu'il voit et qu'il sent comme le peuple s'il ne pouvait, à l'occasion, se sentir authentiquement «peuple ». Mais on peut se demander si son plébéianisme n'exprime pas d'abord un ressentiment d'exclu à l'égard d'une classe dominante qui ne le reconnaît pas comme un des siens et qui lui refusera toujours, dans les occasions décisives, ce qu'il s'obstinera à lui demander (par exemple la consécration de l'Académie). Si le boursier Zola se veut et se fait peuple, s'il encanaille son style et prend à sa manière « la pose à la blouse ", c'est peut-être faute de pouvoir s'embourgeoiser aussi complètement et aussi vite qu'il le voudrait ${ }^{36}$. Les foucades de l'enfant terrible sont encore des gages de sa bonne volonté, au moins culturelle. Ainsi, dans la scène de la visite au Louvre, l'iconoclaste a tôt fait de redonner la parole au cicérone et à l'amateur d'art : tel Goujet entonnant «les Adieux d'Abd el Kader », Zola « ramène le respect»: pas question d'admirer les parquets, pas question de ne pas admirer les Vénitiens, les Hollandais et les Primitifs ${ }^{37}$.

Moyen d'expression privilégié de cette identification-refuge, le style indirect libre est luimême suffisamment ambigu pour permettre la réintroduction de formes détournées d'ethnocentrisme de classe; il suffit pour cela qu'il soit appliqué indifféremment au parler bourgeois et au parier peuple, en dépit de leurs distances inégales à la langue littéraire et à la culture dominante. La suppression des guillemets a pour effet de dérouter le lecteur qui ne sait plus très bien à qui il a affaire, si c'est l'auteur qui parle ou bien ses personnages, si l'auteur fait parler ceux-ci ou s'il les laisse parler, s'il partage leur point de vue ou s'il le donne pour ce qu'il est, et ce d'autant plus que Zola excelle à donner et à reprendre la parole dans la même phrase, à passer de la description "objective » des pratiques au commentaire, et du commentaire à la citation. Mais en s'attachant à présenter les mœurs, les goûts et le parler «peuple » dans leur crudité et dans leur "naïveté » indigènes, Zola les livre sans défense aux jugements du goût dominant; moins il les apprête, plus il semble en respecter l'étrangeté, l'énormité, l'altérité irréductible, et plus il en fait ressortir, sans avoir l'air d'y toucher, l'incongruité, l'inconscience et le ridicule au second degré. Il lui suffit, pour cela, de ne pas accorder le même poids, ni les mêmes privilèges au point de vue des dominants et à celui des dominés ; ainsi la noce de Gervaise ne se voit pas « telle qu'elle est », parce qu'elle ne voit pas qu'elle est vue : c'est en aveugle que Zola la fait défiler devant les représentants du bon goût et de la culture légitime, promeneurs des beaux quartiers, visiteurs habituels du Louvre, dont le point de vue, qui coïncide avec celui de l'auteur et du lecteur cultivé, devient du même coup le seul «vrai » point de vue ${ }^{38}$. De même le style indirect libre permet de donner à voir de l'extérieur le comportement «objectif » des personnages, et de faire entendre simultanément leurs réflexions ou même leurs pensées (par exemple, les noceurs sortent, à la fin du gueuleton, pour aller vomir ; commentaire «indigène »: "quand on a été bien élevé, ça se voit toujours ») ; en construisant ainsi des phrases à double entente et à double détente, dont l'équivoque assez grossière ne demande qu'à être levée, Zola choque sans trop de risques un lecteur à qui il laisse, en dernière instance, le soin d'apprécier à leur juste valeur les «bonnes manières" et le «bon sens " populaires. Un usage un peu trop libre du style indirect libre, et l'extension apparemment impartiale de celui-ci au parler peuple, autorise le sociologue à refaire lui aussi, sous 
couvert d'objectivité scientifique, le coup de l'impersonnalité flaubertienne que le romancier n'ose plus guère se permettre depuis les critiques de Sartre contre Mauriac : à la fois Gygès et Protée, Deus absconditus et Diable boiteux, le sociologue-écrivain intervient quand bon et comme bon lui semble, tout en paraissant n'être que «le greffier de la Nécessité »; lui aussi se paye le luxe de se confondre avec sa création tout en paraissant s'effacer ${ }^{39}$.

Prétendre interdire au sociologue de recourir à la langue littéraire (ou philosophique), le sommer de n'utiliser aucun mot qu'il n'ait au préalable défini et fixé, ne serait pas seulement naïf: le soupçon de non-scientificité que la malveillance conservatrice, aussi sensible aux « obscurités » et aux « équivoques » de l'« écriture » qu'elle semble aveugle au formalisme et au terrorisme de la numéromanie, porte contre les modes d'expression qui s'écartent du pseudo-modèle des sciences exactes (et ce auprès d'un public partagé entre "littéraires» et "scientifiques" par le dualisme institutionnel du système d'enseignement français) pourrait bien être l'équivalent moderne de l'obligation faite aux sciences humaines (et en particulier à l'histoire) par les conservateurs du XIX siècle de s'en tenir à la langue de l'Académie et de "l'honnête homme ", le fétichisme du chiffre remplaçant celui du mot bien sonnant ${ }^{40}$. Mais le sociologue qui «écrit» risque de prendre, avec la pose, la posture de l'écrivain; comme à Zola, il peut lui arriver de se prendre pour Balzac ou pour Flaubert (et, en plus, pour Proust). Cette identification hétéropathique ne risque pas seulement d'infléchir ses choix de recherche et de publication en fonction d'ambitions ajustées sur l'image éblouissante que la littérature donne de la gloire littéraire. L'association mystique du moi et de l'œuvre, unis dans le même culte par la conception romantique du génie, incite d'une manière plus insidieuse à concevoir et à envisager l'œuvre scientifique sur le modèle de l'œuvre littéraire ${ }^{41}$. En se transformant en auteur, le sociologue risque de se laisser imposer la définition romantique de l'autorité, et de se persuader intimement qu'on ne peut trouver l'autorisation d'écrire qu'en soi-même, dans ses dons personnels, dans son charisme et dans son génie (au lieu de la chercher à l'extérieur de soi, dans le travail empirique et l'esprit d'équipe $)^{42}$. À force de s'identifier à l'écrivain, il risque de. perdre le contrôle de ses interprétations et de se condamner à l'intuitionnisme et au subjectivisme; comme Renan, il peut finir par confondre le point de vue du savant avec le coup d'œil infaillible de l'artiste, et en venir à croire que la meilleure façon de pénétrer l'esprit des données est d'en négliger la lettre ${ }^{43}$. À avoir ainsi la berlue, la sociologie risquerait de perdre sa capacité critique. En effet l'ethnocentrisme de classe trouve probablement un bastion inexpugnable dans l'égocentrisme d'auteur; plus encore que les contraintes de l'édition ou que l'anticipation des attentes du public, le fétichisme de l'œuvre personnelle, qui tend à transformer insidieusement les questions de sociologie en questions de goût, risque de maintenir ou de ramener la sociologie en deçà de la rupture avec le point de vue dominant. 


\section{NOTES}

1. E. Renan, Essais de morale et de critique, Paris, Michel Lévy, 1859, p. 129-131.

2. Ibid., p. 122-123.

3. "Sans cette singulière ivresse dont se moquent tous ceux qui restent étrangers à la science, sans cette passion, sans cette certitude que "des milliers d'années devaient s'écouler avant que tu n'aies vu la vie et d'autres milliers d'années attendent en silence"[...] de savoir si tu es capable de faire cette conjecture-là, tu ne posséderas jamais la vocation du savant et tu ferais mieux de t'engager dans une autre voie. Car rien n'a de valeur, pour l'homme en tant qu'homme, qu'il ne peut faire avec passion.» (M. Weber, Le savant et le politique, Paris, 10-18, 1963, p. 63, trad. fr. de Wissenschaft als Beruf, 1919.)

4. Renan, Essais de morale et de critique, p. 115, 132, 133.

5. Ibid., p. 103-109, 117, 118, 131.

6. À propos de Lamennais, Renan oppose au contraire le beau et le vrai, l'« âme» et l'« esprit». «Ce ne fut ni un politique, ni un philosophe, ni un savant, ce fut un admirable poète, obéissant à une muse sévère et toujours irritée [...] La flamme vive et passagère de la passion méridionale n'a rien de commun avec ce feu ardent et sombre, avec cette colère profonde et obstinée qui ne veut pas être adoucie. Il n'y a pas de plus mauvaise disposition pour un philosophe et un critique; il n'y en a pas de meilleure pour un artiste et un poète [...] Le tour absolu des opinions de Lamennais, qui nous a valu tant de pauvres raisonnements, tant de jugements défectueux, nous a valu aussi les cinquantes pages de grand style les plus belles de notre siècle. Jamais plus frappant exemple du partage des dons de l'esprit ne fut offert aux méditations du penseur : Lamennais est inexplicable si l'on n'accorde pas que le même homme peut être à la fois un artiste supérieur, un philosophe médiocre et un politique insensé.» Ibid., p. 145-146.

7. Ibid., p. 131 sq. : «La langue française est arrivée sous ce rapport à un tel degré de perfection, qu'on peut la prendre comme une sorte de diapason dont la moindre dissonance indique une faute de jugement ou de goût.» (Le « diapason» de Renan semble remplir la même fonction que le «gueuloir» de son ami Flaubert : «Pourquoi y a-t-il un rapport nécessaire entre le mot juste et le mot musical ? Pourquoi arrive-t-on toujours à faire un vers quand on resserre trop sa pensée ?» Lettre à Madame Roger des Genettes, 1876.)

8. Renan, Essais de morale et de critique, p. 114. À l'inverse de l'érudit, que son isolement dans le monastère ou la bibliothèque empêche de comprendre tant le passé que son époque, l'historien selon Renan est un «intellectuel engagé»: "C'est la gloire de la grande école à laquelle appartient M. Thierry d'être arrivée à l'histoire par la politique [...] La rénovation des études historiques qui a eu lieu de notre temps est due avant tout à l'initiative de trois ministres, savants sans doute, mais dont la profession particulière n'est pas la recherche de première main : M. Guizot, M. Villemain, M. Cousin, par leurs vues nouvelles sur l'histoire politique, littéraire, philosophique, ont directement fourni autant de textes à la science que le plus laborieux compilateur.» (P.123.)

9. C. Baudelot \& R. Establet, Durkheim et le suicide, Paris, PUF, 1984, p. 82.

10. Sur la théorie de la légitimité culturelle, et sur la tendance des analyses sociologiques « légitimistes» à adopter le point de vue dominant pour décrire les rapports de domination entre les classes, cf. C. Grignon, J.-C. Passeron, Sociologie de la culture et sociologie des cultures populaires, Paris, GIDES, 1983 (rééd. en ligne).

11. L'idée que les goûts et l'« habitus» des personnages se laissent appréhender, sous une forme concrète et objective, dans le décor et dans les attributs emblématiques qui les entourent (ou 
dont ils s'entourent à des fins délibérées de présentation et de stylisation de soi) est déjà présente chez Flaubert : «J'ai renoué connaissance [...] avec M. Cordier, gentleman de ces contrées, ancien sous-préfet de Pont-l'Évêque sous Louis-Philippe, député réac, ex-membre de la parlotte d'Orsay, ex-auditeur au Conseil d'État, jeune homme tout à fait bien, docteur en droit, belle fortune [...] Et des mots: “j'ai renoncé aux vanités, je méprise le monde, je ne m'occupe plus que d'art”. S'occuper d'art! C'est avoir des vitraux de couleur dans son escalier et des meubles en façon LouisXIII.» M. Cordier a commandé des verres artistiques ; il tient à ce que tout chez lui ait « $\underline{\text { un }}$ cachet particulier» (souligné par Flaubert). « Voilà la race commune des gens qui sont à la tête de la Société [...] La médiocrité se couvre d'intelligence, il y a des recettes pour tout, des mobiliers voulus et qui disent : "Mon maître aime les arts. Ici on a l'âme sensible. Vous êtes chez un homme grave !"» (Lettre à Louise Colet, 21, 22août 1853, Correspondance, Paris, NRF, 1980, p. 405 sq., «Bibliothèque de la Pléiade»). Comme il est de règle chez Flaubert, le romancier cultive et récupère les indignations de l'homme. Ainsi « l'autorité de la parole» du docteur Vaucorbeil «se renforce au spectacle des choses environnantes» : « Du diachylum [toile pour les emplâtres], des bandes traînaient sur la cheminée. La boîte chirurgicale posait au milieu du bureau, des sondes emplissaient une cuvette dans un coin, et il y avait contre le mur la représentation d'un écorché» (Bouvard et Pécuchet, Paris, NRF, 1979, p. 763, «Bibliothèque de la Pléiade»). De même, le bric-àbrac dont s'entoure Pellerin, le peintre raté de L'éducation sentimentale, « renforce la puissance de sa parole: on voyait une tête de mort sur un prie-Dieu, des yatagans, une robe de moine; Frédéric l'endossa» (L'éducation sentimentale, même édition, p. 69).

12. Le type idéal des sociologues, tel que le définit Max Weber, s'écarte lui aussi du type moyen, et il est, lui aussi, une «fiction cohérente», une « utopie»; mais il ne prétend en aucune manière exprimer ou représenter la «réalité authentique». Simple «guide pour l'élaboration des hypothèses», il n'a d'autre signification que celle d'un "concept-limite auquel on mesure la réalité pour clarifier le contenu empirique de certains de ses éléments importants et avec lequel on la compare» (M. Weber, Essais sur la théorie de la science, Paris, Plon, 1965).

13. «Ma pensée, en dépit de la vente plus grande que jamais du roman est que le roman est un genre usé, éculé, qui a dit tout ce qu'il avait à dire, un genre dont j'ai tout fait pour tuer le romanesque, pour en faire des sortes d'autobiographies, de mémoires de gens qui n'ont pas d'histoire.» (E. de Goncourt, Réponse à l'enquête de J. Huret sur l'évolution littéraire (1891), cité par P. Cogny, Le naturalisme, Paris, PUF, 1976, p. 52.)

14. Difficultés déjà rencontrées, et analysées, par George Sand : «Si je fais parler l'homme des champs comme il parle, il faut une traduction en regard pour l'homme civilisé, et si je le fais parler comme nous parlons, j'en fais un être impossible, auquel il faut supposer un ordre d'idées qu'il n'a pas [...] Raconte-la-moi [l'histoire du Champi] comme si tu avais à ta droite un Parisien parlant la langue moderne, et à ta gauche un paysan devant lequel tu ne voudrais pas dire une phrase, un mot où il ne pourrait pas pénétrer. Ainsi, tu dois parler clairement pour le Parisien, naïvement pour le paysan. L'un te reprochera de manquer de couleur, l'autre d'élégance.» (G. Sand, François le Champi, Paris, Garnier, 1969, p. 215-217.)

15. «L'épine dorsale [de L'assommoir] est en place avant toute recherche de document [...] Déjà, dans ces notations télégraphiques saisies sur place, et avant même que la première ligne du roman ne fût écrite, l'auteur faisait un choix parmi les éléments que lui fournissait le réel, ne gardant que les détails utiles au découpage du récit, et ceux qui cristallisaient, à ses yeux, la tristesse de l'habitat ouvrier: les persiennes noires, le ruisseau sous le porche, les portes uniformes.» (H.Mitterand, «Étude de L'assommoir», RM, II, p. 1550 : pour Les Rougon-Macquart, les références renvoient à l'édition de la NRF, «Bibliothèque de la Pléiade», avec indication du tome, suivie de la pagination.) Cf. aussi H.Massis, Comment Émile Zola composait ses romans, Paris, Fasquelle, 1906, p.327-328: «Le romancier expérimentateur doit (d'après Zola) "voir, comprendre, inventer" ; lui, Zola, invente, voit, puis donne aux faits observés "la flamme de sa nature et l'arrangement de son goût" [...] Si l'observation d'Émile Zola n'a rien d'un naturaliste, 
sa documentation n'a rien d'un savant: scrupuleuse jusqu'à la manie, abondante jusqu'à la prolixité, elle est, le plus souvent, sans portée et superficielle; elle demande la vérité à des témoins presque toujours suspects : aux journaux, aux revues, aux manuels.»

16. « Ne pas flatter l'ouvrier et ne pas le noircir. Une réalité absolument exacte. Au bout la morale se dégageant elle-même. Un bon ouvrier fera l'opposition; ou plutôt non, ne pas tomber dans le Manuel» (É. Zola, « Dossier de L'assommoir. Ébauche», cité in H.Massis, Comment Émile Zola composait ses romans, p. 101.

17. Ibid.,p.100, 101.

18. Ibid., p. 101 sq.

19. D. Poulot, Question sociale. Le Sublime ou le travailleur comme il est en 1870 et ce qu'il peut être, Paris, Librairie internationale, 1870. Sur les emprunts de Zola à D. Poulot, cf. H.Massis, Comment Émile Zola..., p. 528 ; voir aussi P. Cogny, «Zola et Le Sublime de Denis Poulot», Cahiers de l'Association internationale des Études françaises, 24, mai 1972, p. 113-129; H.Mitterand, «Étude de L'assommoir», RM, II, p. 1532-1568.

20. "L'hypotypose peint les choses d'une manière si vive et si énergique, qu'elle les met en quelque sorte sous les yeux, et fait d'un récit ou d'une description, une image, un tableau, ou même une scène vivante» (P. Fontanier, Les figures du discours, Paris, Flammarion, 1968, rééd., p. 390).

21. «Le sujet est pauvre : il faudrait voir à le faire alors d'une vérité telle qu'il soit un miracle d'exactitude» (É. Zola, « Dossier de L'assommoir. Ébauche», cité in H.Massis, Comment Émile Zola..., p. 101 sq.). Sur les problèmes d'écriture que la perspective misérabiliste pose au romancier comme au sociologue, et sur la manière dont Zola se tire d'affaire en décrivant non le dénuement, techniquement indescriptible, mais la privation, cf. C. Grignon, « Sociology of taste and the realist novel : representations of popular eating in E. Zola», Food and Foodways, 1 (2), 1986, p. 117-160.

22. Renan, Essais de morale et de critique, p. 126, 132.

23. Ce n'est sans doule pas un hasard si les sociologues tendent eux aussi à privilégier, parmi les aliments effectivement surconsommés par les classes populaires, ceux dont les propriétés matérielles et sensorielles (telles du moins qu'elles sont socialement perçues) évoquent les propriétés que l'imaginaire dominant prête au peuple et aux «masses» -en particulier les aliments "lourds», " consistants», "bourratifs», "indifférenciés»,etc. Cf. C. et C. Grignon, "Styles d'alimentation et goûts populaires», Revue française de Sociologie, 21, 1980, p. 531-569: «Les classes populaires risquent fort de se trouver associées automatiquement aux plats et aux goûts qui se situent, comme elles, du côté de l'indifférencié et du magma, du bouilli plutôt que du rôti, des sauces plutôt que des grillades, du mijoté plutôt que du saisi,etc. ; là encore, on tendra à privilégier les préparations les plus évocalrices et les plus adéquates, par exemple les pommes de terre bouillies plutôt que les frites, les hachis ou le pot-au-feu plutôt que les côtes de porc, les compotes plutôt que les tartes ou les tartines.»

24. Sur les insuffisances et sur les archaïsmes de la documentation de Zola, notamment en ce qui concerne le parler populaire, cf. A.Lanoux, Bonjour Monsieur Zola, Paris, Grasset, 1978, p. 145-146 : «L'argot qu'adorait Hugo est lyrique et prodigieux, langue d'un Homère à cent mille bouches. Celui de Balzac est dosé, rare, exact. Celui de Zola restera plaqué. Il n'utilise pas systématiquement le parler vrai des barrières et des faubourgs parce qu'il n'a pas le temps de l'apprendre. Aussi l'argot de L'assommoir sera-t-il en retard d'une trentaine d'années sur celui qu'auraient dû parler ses personnages. Même le titre surprit les gens de la Chapelle. On ne disait pas un "assommoir", à Barbès, mais un bistrot. Le mot est maintenant académique.» De même, à propos de Germinal, A.Lanoux note que «Zola n'est pas entré dans le patois savoureux de ses gens du Nord [...] Ses héros parlent peuple sans parler ch'timi» (souligné par Lanoux, p. 210).

25. Le volontarisme et l'égocentrisme de Zola se livrent sans détour dans les Notes préliminaires qu'il rédige en 1868 («Sur la marche de l'œuvre», « Sur la nature de l'œuvre», « Différences enlre 
Balzac et moi»,etc.) : « On sent toujours chez lui l'instinct de se faire centre et de rapporter tout à soi. Il est de tempérament conquérant; il a une idée fixe : dominer [...] Mon œuvre, pense-t-il, sera une conformité à cette science. Je vais peindre l'homme physiologique. Ma formule est là et d'elle va naître un nouvel art, une nouvelle littérature, qui sera ma littérature à moi, mon art à moi. Le naturalisme, ce sera mol, moi tout seul». H.Massis, Comment Émile Zola..., p. 9.

26. Cf. Goncourt, Journal, 27août 1870 : «Zola [...] m'entretient d'une série de romans qu'il veut faire, d'une épopée en dix volumes de l'histoire naturelle et sociale d'une famille, qu'il a l'ambition de tenter [...] Il me dit : après les analyses des infiniments petits du sentiment, comme cette analyse a été tentée par Flaubert dans Madame Bovary, après l'analyse des choses artistiques, plastiques et nerveuses, ainsi que vous l'avez faite, après ces œuvres bijoux, ces volumes ciselés, il n'y a plus de place pour les jeunes, plus rien à faire ; plus à constituer, plus à construire un personnage, une figure : ce n'est que par la quantité des volumes, la puissance de création qu'on peut parler au public.»

27. É. Zola, "Premier plan remis à l'éditeur A.Lacroix», cité in H.Massis, Comment Émile Zola..., p. 62-74.

28. Cf. H. Massis, ibid., p. 8 : « [Zola] se fit une étude d'un Traité de l'hérédité naturelle du Dr Lucas. Il y rencontra la théorie qu'il lui fallait et en tira quelques affirmations, très simples, énergiques, tenaces». Prises « avec un sérieux et une gravité de profane» (Massis), les notes de lecture de Zola ressemblent beaucoup -mais au premier degré- à celles que Flaubert prenait pour Bouvard et Pécuchet ou pour ses «sottisiers»: «trait originel : gros nez des Bourbons»; «les enfants supérieurs naissent généralement de parents simples et vice versa»; «il arrive que le père donne le physique et la mère le moral, mais souvent aussi le contraire»; «le produit d'un vieux mâle ressemblera à la jeune femelle. Les jeunes mariés ont des filles»,etc.

29. Comme le note Louis Chevalier (Classes laborieuses et classes dangereuses, à Paris pendant la première moitié du XIX ${ }^{e}$ siècle, Paris, Plon, 1969, p. 477), le peuple de Paris «s'incarne, jusqu'aux dernières décades du XIX ${ }^{\mathrm{e}}$ siècle, en quelques types, choisis en fonction d'apparences pittoresques et qui semblent immuables, en dépit de l'évolution du temps». De ce point de vue, les naturalistes sont les héritiers d'une tradition qui va de Restif de laBretonne et de Louis-Sébastien Mercier à Champfleury et à Duranty, leurs prédécesseurs immédiats de la "Bohème réaliste», en passant par les écrivains et les dessinateurs de la génération romantique, comme Henri Monnier, Daumier, Gavarni, et, à l'occasion, Balzac ou Hugo. Comme eux, ils mettent l'accent sur les plaisirs ouvriers plutôt que sur le travail, et font passer au premier plan les personnages populaires les plus voyants et les plus bruyants (ceux qui occupent la rue et qui sont également surreprésentés dans l'estampe, dans la presse et dans les archives de la police). Eux aussi sont en retard sur les transformations des mœurs, du langage et des conditions de vie; dans sa définition pittoresque, le typique est d'abord le stéréotypé et le convenu, ce que l'observateur extérieur reconnaît pour avoir appris à le reconnaître, pour l'avoir déjà lu ou déjà vu.

30. « Dossier de L'assommoir», in H.Massis, Comment Émile Zola..., p. 227, 231, 239, 254, 260, 291. Les notes de Zola portent encore la marque de l'anti-ouvriérisme qui suit la Commune, et dont témoignent, entre autres, les Contes du lundi d'Alphonse Daudet. L'ouvrier Arthur préfigure à la fois Lantier et Bijard, la brute alcoolique de L'assommoir : tous les samedis, il « bat sa femme et boit sa paye»; le dimanche, il "fait l'aimable, le bel esprit», on dirait "un de ces ouvriers modèles qui suivent les cours du soir»; il « déclame des bouts d'idées ramassées un peu partout, sur les droits des ouvriers, la tyrannie du capital» et chante "les Hirondelles», de Béranger, " avec le sentimentalisme bête de l'ouvrier»; "sa pauvre femme, attendrie par les coups de la veille, le regarde avec admiration» (A.Daudet, Arthur, première publication dans Le Soir du 28 nov. 1871).

31. Francisque Sarcey, article paru dans Le Gaulois, 8 fév. 1870, cité par H.Massis, Comment Émile Zola..., p. 189-198. 
32. Dans le plan primitif du chapitreIII, la noce de Gervaise devait aller faire une promenade (sociologiquement moins improbable) sur les fortifications (H. Massis, ibid., p. 215).

33. «À l'inconvenance du fond, M. Zola, par surcroît, s'applique à joindre la grossièreté de la forme[...] On peut dire [...] que L'assommoir était un roman de mœurs populaires, ou, plus exactement populacières, et qu'après tout, le langage qui s'y parlait, nous en avions, de ci, de là, du côté du boulevard de la Villette et du boulevard des Gobelins, entendu les mots bourdonner à notre oreille. Il y avait d'ailleurs accord de la forme et du fond, et la brutalité des procédés y convenait très étroitement à la vulgarité des mœurs [...] Toute la question, mais une question capitale, d'où dépendait l'estime à faire de la vraie valeur de M. Zola, n'était que de savoir ce qu'il adviendrait de ces illusions de talent quand il changerait de milieu. Il en est advenu Pot-Bouille, et c'est presque assez dire [...] Nous avons compris ce que voulaient dire ces grossièretés inutiles et, si l'on veut bien me permettre une seule fois la seule expression qui convienne, ces ignobles coups de gueule de L'assommoir et de Nana [...] Si M. Zola manque de goût et d'esprit, comme s'il manque de finesse psychologique, c'est que M. Zola manque de sens moral.» (F.Brunetière, « À propos de Pot-Bouille», Revue des Deux-Mondes, 15mai 1882, p. 454-465.)

34. Sur « l'inconscient» du style indirect libre, et sur sa capacité à «transposer l'objectif dans le subjectif», cf. C. Bailly, "Le style indirect libre en français moderne», Germanisch-Romanische Monatsschrift, IV, 1912, p. 549-556 et 597-606.

35. «Zola fait passer l'argot du style direct au style indirect, l'étendant jusqu'aux endroits où il rapporte non point les dires, mais les pensées de ses gens, qui pensent partie en argot. Puis, comme il n'est guère de scène que l'auteur ne puisse considérer du point de vue de ses personnages, qu'il ne puisse présenter telles que ceux-ci les voient et les présenteraient euxmêmes, la langue du milieu devient celle de l'auteur.» (F.Brunot, cité in H.Massis, Comment Émile Zola..., p. 331.)

36. Le cas de C. Péguy, lui aussi orphelin et boursier, fournit un autre exemple de "plébéianisme». Parce que tout, dans la relation qu'il entretient avec les représentants de son milieu d'arrivée (et en particulier avec ses condisciples de l'École normale supérieure), lui fait sentir qu'il ne sera jamais vraiment un des leurs, Péguy se voit, se sent et en définitive se veut peuple, et plus peuple que nature, paysan ou plutôt, comme il dit, « pesan» : «Il ne faut pas nous le dissimuler, Halévy, nous appartenons à deux classes différentes [...] Quoi que vous en ayez, quoi que vous fassiez, quoi que vous y mettiez et dans le vêtement et dans tout l'habitus, et dans la barbe et dans le ton, et dans l'esprit et dans le cœur [...] Moi aussi j'espérais qu'un jour j'aurais cette suprême distinction, cette finesse, cette suprême élégance d'un (Marcel) Mauss (pas le marchand de vin), la diction, la sévère, l'impeccable, l'implacable diction, la finesse d'un "Boiteà-fiches" [...] À présent il faut me rendre. Il faut que je capitule [...] Cette élégance de Mauss il faut y renoncer. Ce fin du fin, ce fin profil, ce regard noble, assuré, nullement voyou, ce langage fleuri, ces lèvres amènes, ce veston démocratique mais fin, démocratique mais sobre [...] ce long pantalon sociologue [...] ce fin parler haut allemand, ce teint de lys et de roses, il y faut renoncer [...]. Moi vous le savez. Voyons, vous le savez bien [...]. Ce que je suis, Halévy, il suffit de me voir, il suffit de me regarder, un instant, pour le savoir [...] En moi, autour de moi, dessus moi, sans me demander mon avis, tout conspire, au-dessus de moi, tout concourt à faire de moi un paysan non point du Danube, ce qui serait de la littérature encore, mais simplement de la vallée de la Loire, un bûcheron d'une forêt qui n'est même pas l'immortelle forêt de Gastine, puisque c'était la périssable forêt d'Orléans, un vigneron des côtes et des sables de Loire. Déjà je ne sais plus quoi dire, ni même comment me tenir dans ces quelques salons amis, où j'allais quelque fois.» À sa biographie réelle d'écrivain et d'intellectuel, le directeur des Cahiers de la Quinzaine substitue la biographie imaginaire d'un "gars Péguy» tôt supplanté par l'élève Péguy : comme ses ancêtres mythiques, « les patients aïeux noueux comme les ceps» qui «n'en ont pas eu pour longtemps à reconquérir sur le monde bourgeois, sur la société bourgeoise, leur petit-fils indigne, buveur d'eau, en bouteilles», Péguy sera " un vieux rompu, un vieux tordu, un vieux moulu, un vieux 
tortu [...]. On dira : c'est le père Péguy qui s'en va» (C. Péguy, La république, notre royaume de France , Paris, Gallimard, 1946, p. 261 sq.)

37. C'est sans doute dans ses souvenirs scolaires que le boursier d'origine "modeste» peut trouver le plus facilement les « racines» et l'« identité» populaires qu'il est tenté d'opposer aux rebuffades que lui réserve son milieu d'aspiration. Fréquent chez les auteurs naguère en usage à l'école primaire, le thème $\mathrm{du}$ repas populaire se prête tout particulièrement à cette reconstitution, la scène lue en classe tenant lieu de scène vécue (ce qui pourrait contribuer à expliquer le passage direct de la littérature à la sociologie de certains clichés, comme la soupe maternelle -souvenir d'H. Malot ?-, l'atmosphère patriarcale du repas paysan -F. Mistral ?- ou l'ambiance bon enfant des tablées populaires). Sur le "masque plébéien» dont se parent à l'occasion les théories de la domination les plus enracinées dans un «socio-centrisme de classe supérieure», cf. J.-C. Chamboredon, « Réponse à MM.Boudon (B1) et Bourricaud (B2), auteurs du Dictionnaire», Revue française de Sociologie, 25, 1984.

38. «Peu à peu, pourtant, le bruit avait dû se répandre qu'une noce visitait le Louvre; des peintres accouraient, la bouche fendue d'un rire: des curieux s'asseyaient à l'avance sur des banquettes pour assister commodément au défilé ; tandis que les gardiens, les lèvres pincées, retenaient des mots d'esprit» (RM, II, p. 446).

39. Sur l'usage risqué que la sociologie «légitimiste» des goûts fait du style indirect libre, cf. C. Grignon et J.-C. Passeron, Sociologie de la culture et sociologie des cultures populaires, p. $124 \mathrm{sq.}$

40. Héritière de la critique du pédantisme, la dénonciation rituelle du «jargon» pourrait bien n'être que l'expression honorable des censures que la bienséance conservatrice prétend imposer à la sociologie critique de crainte que celle-ci, installée dans ses mots, cesse de mettre les formes ou, pire, trouve des formes adéquates à son propos. Même s'il a tendance à se transformer lui aussi en "langue de bois», le «jargon» des sociologues mérite au moins la considération populiste qu'on accorde aux argots de métier ou de minorités.

41. Sur l'œuvre comme viatique en ce monde, cf. E. Renan : «Il ne sera question que de ce qu'il [A. Thierry] a aimé et de ce qui lui a fait supporter la vie, je veux dire son œuvre». Le fétichisme de l'œuvre apparait tout particulièrement dans la littérature édifiante sur les derniers moments de l'écrivain, dont l'exemple le plus connu est sans doute la scène de la mort de Bergotte, avec ses livres, « symboles de sa résurrection», qui veillent sur lui trois par trois, « comme des angos aux ailes éployées», dans le plus pur style funéraire sulpicien.

42. Ainsi «quelques lambeaux de textes, médiocrement entendus quant à la lettre, révélèrent plus de choses à $\mathrm{M}$. de Chateaubriand que n'en avait appris aux érudits de la vieille école l'étude la plus consciencieuse des monuments du Moyen Âge et de l'Antiquité» (Renan, Essais de morale et de critique, p. 123). Témoin encore la parabole d'Augustin Thierry devenu presque aveugle et voyant d'autant plus clair avec les yeux de l'esprit (ou de l'âme) : «Parcourant avec M. Fauriel le Midi de la France, et n'ayant tout juste de vue que ce qu'il fallait pour se conduire, il retrouvait, en présence des ruines, toute sa facilité de lecture. Son œil, si incertain dans les circonstances ordinaires, était alors d'une merveilleuse promptitude : aucune des lignes principales, aucun trait caractéristique ne lui échappait» (p.115).

43. Le romancier classique, au contraire, recourt à des « expédients [...] pour se donner l'air de ne pas fabuler -manuscrit trouvé par hasard, relation d'un témoin oculaire ou d'un personnage mêlé de près aux événements, confession du héros lui-même, échange de lettres, relation d'un fait divers ou d'une aventure réellement arrivée- les procédés en eux-mêmes n'importent guère, tous sont bons, paradoxalement, pourvu qu'ils entretiennent l'illusion d'un auteur inconnu, dont le vrai ne serait en somme que l'exécuteur.» (M. Robert, Livre de lectures, Paris, Grasset, 1976, p. 84.) 